\title{
EL ESTUDIO DE LA CERÁMICA EN LA RECONSTRUCCIÓN DE LAS HISTORIAS LOCALES. EL SUR DE LA QUEBRADA DE HUMAHUACA (JUJUY, ARGENTINA) DURANTE LOS DESARROLLOS REGIONALES E INCAICO
}

\author{
THE ROLE OF CERAMIC STUDIES IN THE RECONSTRUCTION OF LOCAL \\ HISTORY. THE SOUTHERN PORTION OF THE HUMAHUACA GORGE (JUJUY, \\ ARGENTINA) DURING THE LATE INTERMEDIATE AND INKA PERIODS
}

\author{
María Beatriz Cremonte ${ }^{1}$
}

\begin{abstract}
En este trabajo se comentan los resultados obtenidos del análisis petrográfico de pastas cerámicas pertenecientes a vasijas que proceden de asentamientos ubicados en los sectores centro, sur y borde oriental de la quebrada de Humahuaca. En la muestra seleccionada se incluyeron los tipos locales que caracterizan a las ocupaciones Humahuaca y Humahuaca Inka, así como otros con rasgos característicos de la alfarería chaqueña más oriental, excavados en la pequeña guarnición incaica El Cucho de Ocloyas. Las variaciones registradas en las pastas y en algunos atributos morfológicos y decorativos cerámicos, así como en los patrones constructivos y mortuorios, permiten plantear diferencias en los registros arqueológicos de las unidades sociopolíticas desarrolladas en el sector meridional y central de la quebrada de Humahuaca (ca. 1.280-1.536 d.C.). Se plantean estas diferencias materiales como referentes de identidad grupal o social que podrían correlacionarse con los territorios políticos de los Tilcaras (en el centro) y de los Tilianes (al sur), citados en fuentes documentales del siglo XVI como descendientes de antiguos señoríos quebradeños.
\end{abstract}

Palabras claves: Andes Centro Sur, Noroeste Argentino, cerámica, análisis de secciones delgadas.

This paper presents results obtained from petrographic analyses from ceramic vessels belonging to the Humahuaca valley settlements located in central,eastern and southern portions of the Humahuaca gorge. The sample included local ceramic types from Humahuaca and Humahuaca-Inka occupations, and others corresponding to a "chaco tradition" ceramic from El Cucho de Ocloyas, a small Inka fort. The variations detected in ceramic content, as well as morphological and decorative attributes along with architectural and mortuary features are cited as evidence for differentiation between the sociopolitical units within the middle and southern areas (ca. 1,280-1,536 A.D.) in the Quebrada de Humahuaca. These material differences are associated with group or social identities most likely related to the Tilcaras (middle portion) and the Tilianes (to the South) political territories. Both of these groups were mentioned in ethnohistoric documents sources as descendants from the old Humahuaca valley chiefdoms.

Key words: South Central Andes, Northwestern Argentina, ceramics, thin section analysis.

La quebrada de Humahuaca es un área extranuclear andina enclavada en el centro de la provincia de Jujuy. Este estrecho valle andino de $180 \mathrm{~km}$ de longitud y sus importantes quebradas transversales fueron "corredores" de articulación económica, social y cultural a lo largo de su historia. El sector sur de la quebrada se extiende desde la localidad de Volcán (235' S y 65²6' W) hasta el valle de Jujuy (Figura 1). La zona de Volcán marca el límite entre los semidesiertos del norte (la prepuna) y la vegetación húmeda subtropical (yungas). Una característica distintiva de este sector sur de la quebrada de Humahuaca es su proximidad con las yungas ubicadas en los valles sudorientales (cuencas de los ríos Tiraxi y Tesorero), donde se detectaron ocupaciones desde el 800 d.C. hasta por lo menos el 1.440 d.C. Las mismas reflejarían el interés en la ocupación y explotación de los recursos de yungas por parte de los grupos asentados en Volcán (Cremonte y Garay de Fumagalli 1998).

Hacia fines del siglo X (inicio del período de los Desarrollos Regionales del NOA) en la quebrada de Humahuaca ocurrieron cambios sociopolíticos, económicos y demográficos como los acaecidos en otras regiones del NOA y surandinas. Surge un patrón de instalación de poblados

\footnotetext{
1 CONICET-Universidad Nacional de Jujuy, Instituto de Geología y Minería. Av. Bolivia 1661, (4.600) Jujuy. cremonte@idgym.unju.edu.ar
} 


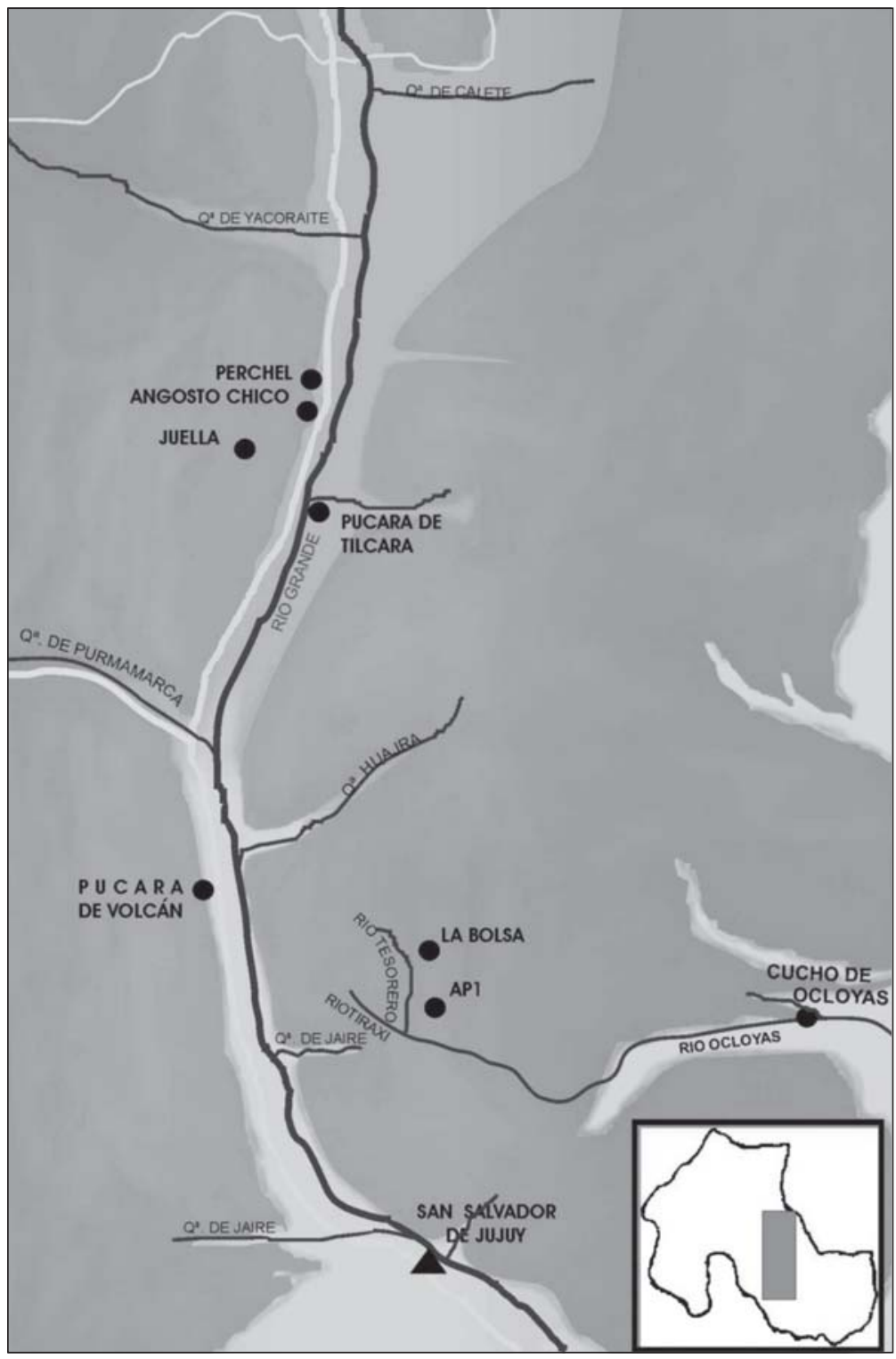

Figura 1. Quebrada de Humahuaca (centro, sur y borde suroriental). Las muestras cerámicas estudiadas provienen de los sitios indicados.

Humahuaca Valley (middle, south and southeastern borders), pointing out sites from which the ceramic samples were derived.

conglomerados, emplazados en lugares altos y fácilmente defendibles (pukaras), evidenciado por la profusión, magnitud y visibilidad de los asentamientos.

El dominio Inka se reconoce en construcciones emplazadas en los sitios de los Desarrollos Regionales, en la construcción de una red vial troncal y de caminos secundarios que conectaban con la puna y los valles orientales, en tambos asociados a los mismos, en la explotación de áreas de producción agrícola para el estado, en el énfasis en la explotación minera y en la instalación de enclaves en las yungas y en la frontera oriental.

Las vasijas pintadas con motivos geométricos en Negro sobre Rojo representan el estilo emblemático de las sociedades de los Desarrollos Regio- 
nales que posteriormente incorporaron atributos incaicos, demostrando la presencia estatal y su afiliación al imperio.

Los conjuntos fechados a partir de 1.280 d.C. "fases Sarahuaico y Pukara" (Nielsen 1997) reflejan la formación y consolidación de este estilo que caracterizara a la cerámica de las sociedades de la quebrada de Humahuaca hasta los fines de la época prehispánica. Los motivos corresponden en su gran mayoría a bandas reticuladas, triángulos negros o reticulados, espirales, "manos o alas", cruces, dameros, etc., organizados en diferentes configuraciones. Las decoraciones pueden aparecer tanto en el interior de pucos y escudillas como en el exterior y bordes internos de cántaros y ollas de cuerpos globulares y bordes evertidos. Otros tipos y estilos locales asociados son los pucos Poma Negro sobre Rojo, decorados externamente con bandas gruesas curvilíneas, los pucos con Interior $\mathrm{Ne}$ gro Pulido, las ollas Angosto Chico Inciso (ACHI) que presentan hileras de incisiones en sus cuellos y las Corrugadas (estas últimas directamente asociadas al momento incaico).

Los estilos de distribución regional en gran medida encubren y desdibujan las variantes locales en lugares que, aunque cercanos, pudieron tener historias diversas. En razón de ello, si se considera que algunas de sus características pueden reflejar diferencias entre las entidades sociales de una región, es necesario no solamente registrar variaciones mínimas en las formas y en las decoraciones, sino también discriminar aspectos tradicionales de manufactura (revelados fundamentalmente a través de las pastas). Las investigaciones reiniciadas en el Pucara de Volcán y las prospecciones en las Yungas del borde oriental permitieron detectar idiosincrasias locales en la manufactura alfarera, así como en otros elementos de los registros arqueológicos y se identificaron vasijas de procedencia no local que plantean la participación de estos grupos en redes de interacción con sociedades que vivían en otros ambientes (Cremonte y Garay de Fumagalli 1997, 1998; Garay de Fumagalli 1998).

El sector meridional de la quebrada de $\mathrm{Hu}$ mahuaca es una zona privilegiada, donde a cortas distancias se obtienen abundantes recursos de yungas: pieles vistosas, maderas duras, miel, tintes vegetales, plantas medicinales, plumas multicolores, nueces, recursos de caza y Anadenanthera collubrina var. cebil (cebil de especie alucinógena). Asimismo, en los espacios abiertos de la forma- ción de Bosque Montano se obtiene buena producción de papa semilla y maíz que pueden sembrarse en forma anticipada, permitiendo la producción de un doble ciclo de siembra (michka).

En este trabajo se comentan las diferencias registradas entre las cerámicas del centro y sur de la quebrada de Humahuaca, poniendo énfasis en los últimos análisis petrográficos de las pastas para discutir la existencia de una probable frontera entre el sector central y sudoriental de la quebrada de Humahuaca (Cremonte 2001; Cremonte y Solís 1998). En el marco de un análisis contextual se espera que las diferencias registradas a nivel cerámico se reiteren en otros elementos de la cultura material. Lamentablemente en el sector sur y oriental la falta de preservación de artefactos confeccionados en madera, cestería, textiles, etc., impide encarar estudios comparativos similares, razón por la cual, y como ocurre en el NOA, la alfarería sigue siendo el mejor referente material de las prácticas sociales prehispánicas.

\section{El Poblamiento Prehispánico Tardío en el Sector Sur y Valles Orientales}

El Pucara de Volcán (Figura 2) es el único poblado prehispánico de gran envergadura conocido en el sector sur de la quebrada de Humahuaca y revela una historia ocupacional desde aproximadamente el siglo XIII hasta el siglo XVI (Garay de Fumagalli 1998). Se trata de un extenso poblado elevado de aproximadamente 7 ha que se extiende a lo largo de una peneplanicie a $150 \mathrm{~m}$ por encima del eje del río Grande y a $1.900 \mathrm{msm}$. A su vez, se relaciona espacialmente con otros sitios contemporáneos conformando un sistema de asentamiento.

De los casi 600 recintos que conforman el área residencial el 95\% presenta ángulos redondeados, una clara diferencia con los sitios del sector central y norte de la quebrada. Este patrón constructivo se reitera en la quebrada del Toro (borde de Puna salteño) y en sitios del valle Calchaquí.

El Pucara de Volcán posee un camino que lo divide longitudinalmente, un cementerio segregado, tumbas asociadas a un montículo artificial en un amplio espacio de participación comunitaria y también enterratorios en el área residencial. Con relación a estos últimos, las cámaras funerarias fueron construidas en los "patios" de las viviendas y no en los recintos menores de las mismas que es lo común en los sitios del sector central de la que- 


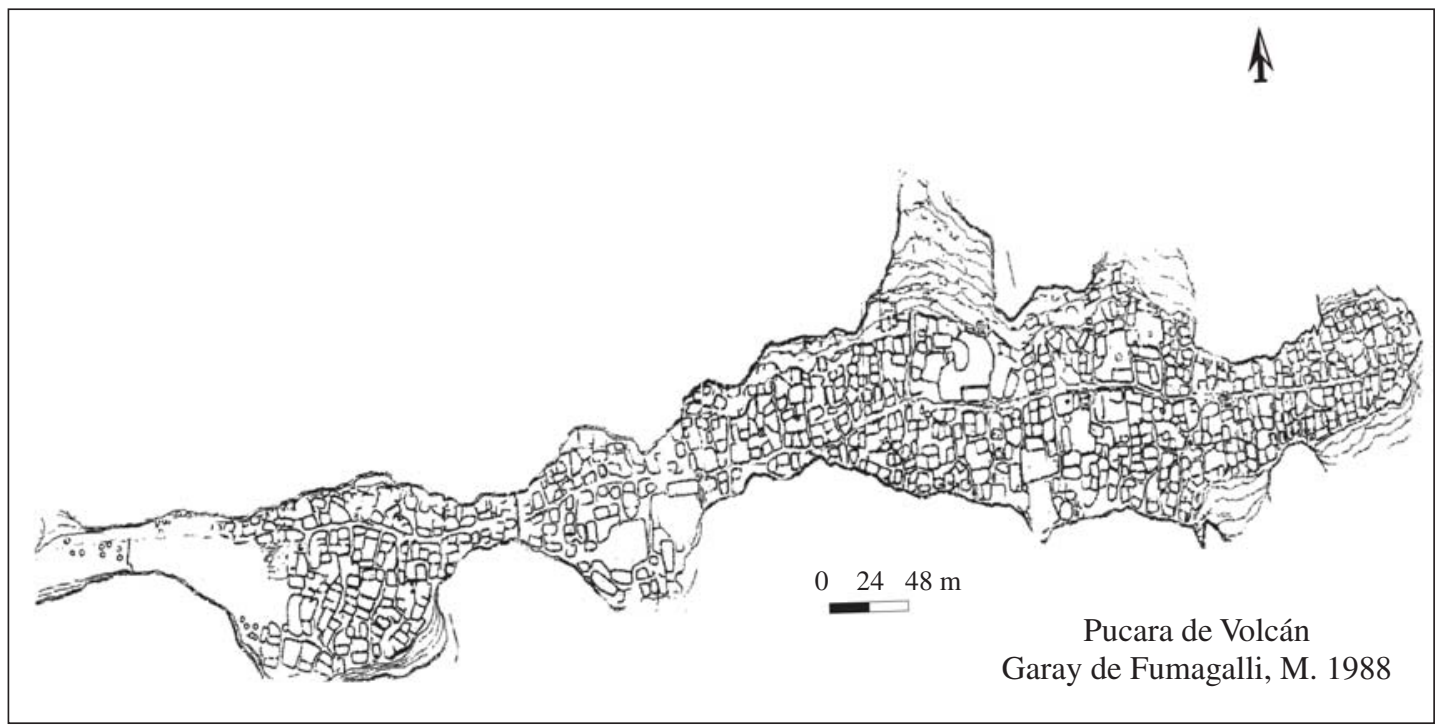

Figura 2. Plano del asentamiento Pucara de Volcán.

Layout of the Pucara de Volcán.

brada. Lamentablemente no se puede avanzar en el conocimiento de las prácticas mortuorias, ya que no quedan en el sitio tumbas sin intervención y, además, se desconocen los datos contextuales de las excavaciones realizadas previamente. Sin embargo, estudios bioarqueológicos comparativos entre sitios de la quebrada de Humahuaca y sugieren que este asentamiento, junto con Ciénaga Grande (en la quebrada de Purmamarca) y Pucara de Tilcara (Departamento Tilcara), se diferencian en su variabilidad genética, probablemente debido al aporte migratorio de otros grupos de regiones limítrofes (Cocilovo et al. 2001).

\section{Características Diferenciales de las Pastas Cerámicas del Sector Meridional y Central de la Quebrada de Humahuaca}

Las 67 secciones delgadas analizadas corresponden a cerámicas de tipos locales, seleccionadas sobre la base de caracterizaciones previas en lupa binocular, procedentes de sitios del sector meridional y borde oriental (Pucara de Volcán, La Bolsa, Sitio Álvarez Prado 1 [AP1], El Cucho de Ocloyas) y del sector central (Pucara de Tilcara, Perchel, Juella y Angosto Chico).

Para el estudio petrográfico comparativo las muestras fueron ordenadas en dos series. La Serie 1 reúne a los tipos Ordinarios, Alisados, ollas
ACHI, vasijas Corrugadas, Unguiculadas y con improntas de Cordelería. La Serie 2 incluye a los tipos Humahuaca y Humahuaca Inka: vasijas decoradas en Negro sobre Rojo y lisas con engobe rojo, pucos con Interior Negro, pucos Poma Negro sobre Rojo, Rosados pulidos y aríbalos.

Del análisis de agrupamiento (cluster analysis) aplicado a los porcentajes (medidos por point counter) de las inclusiones no plásticas de las 35 pastas de la Serie 1 (cuarzo, feldespato potásico, plagioclasas, cuarcitas, filitas y pizarras, tiesto molido, granito y basalto porfírico) se obtuvo un fenograma en el que pueden discriminarse varios agrupamientos (Figura 3). Del análisis se desprende que: (a) las pastas de los subgrupos 14 a 12 y 31 a 15: Ordinarios y ollas ACHI de la variedad A-B, procedentes de los sitios del sector central de la quebrada de Humahuaca, son similares entre sí y se diferencian de las del Pucara de Volcán y sitios del borde oriental. Presentan abundantes filitas y pizarras (20 a 40\%) en comparación con los otros componentes, cuarzo, feldespato potásico y cuarcitas. Estas pastas no contienen tiestos molidos agregados, litoclastos de granito ni de basalto porfírico. Las ollas ACHI de la variedad A-B son las predominantes en el sector central y sus similitudes con las pastas de las vasijas Ordinarias indican una misma tecnología de manufactura y el carácter local de las mismas. A su vez, si bien estas pas- 
tas poseen similitudes con las de los tipos Ordinarios, Naranja Alisados y Unguiculados del Pucara de Volcán y de AP1 (subgrupo 18 a 5), estas últimas presentan un contenido de cuarcitas notoriamente más elevado (9 a 21\%); (b) las pastas ACHI de las variedades $\mathrm{C}$ y D del Pucara de Tilcara con abundante cuarzo y feldespato potásico (11 a 27\%), cuarcitas y/o litoclastos de granito y que pueden presentar tiesto molido son similares a pastas ACHI, Corrugadas, Alisadas y Ordinarias del Pucara de Volcán y de sitios del borde oriental (subgrupos 35 a 23, 32 a 17 y 19 a 1). Estas pastas son muy raras en el sector central de la quebrada de Humahuaca y corresponden a vasijas pequeñas indicando que se trata de manufacturas no locales procedentes del área meridional; (c) las pastas de fragmentos Ordinario, ACHI y Corrugado de El Cucho de Ocloyas (subgrupo 26 a 10) se caracterizan por presentar litoclastos de basalto porfírico, así como abundante cuarzo, tiesto molido y granito, mientras que el contenido de filitas y pizarras es muy bajo. Cuerpos de rocas basálticas afloran en la localidad de Ocloyas y también hacia el este, en la serranía de Santa Bárbara. La arena de un arroyo que corre frente al sitio presenta los mismos pórfi- ros basálticos, junto con otros componentes de formas y tamaños análogos a los de las pastas cerámicas. Puede plantearse, entonces, que las cerámicas de El Cucho de Ocloyas con basalto porfírico fueron fabricadas en el sitio, habiéndose utilizado como antiplástico una arena local como la descrita, o bien, proceder de las sierras de Santa Bárbara, pero siempre dentro del ámbito de las sierras subandinas; (d) las pastas del subgrupo 29 a 2 (Alisados, ACHI, Corrugado, Unguiculado y con improntas de Cordelería de El Cucho de Ocloyas, Pucara de Volcán y AP1) presentan abundante cuarzo y feldespato potásico ( 9 a $15 \%$ ) y basalto porfírico (10 a 24\%). La presencia de pastas como las de El Cucho de Ocloyas en dos fragmentos alisados de AP1 y La Bolsa así como en otros dos fragmentos ACHI del Pucara de Volcán, indica que estas vasijas procederían de El Cucho de Ocloyas o de algún otro sitio próximo, ya que estos basaltos porfíricos están ausentes en la quebrada de Humahuaca y alejados del lugar donde se emplazan AP1 y La Bolsa.

Los resultados del análisis petrográfico de las 32 secciones delgadas de tipos Humahuaca y Humahuaca Inka (Serie 2) mostraron como tendencia

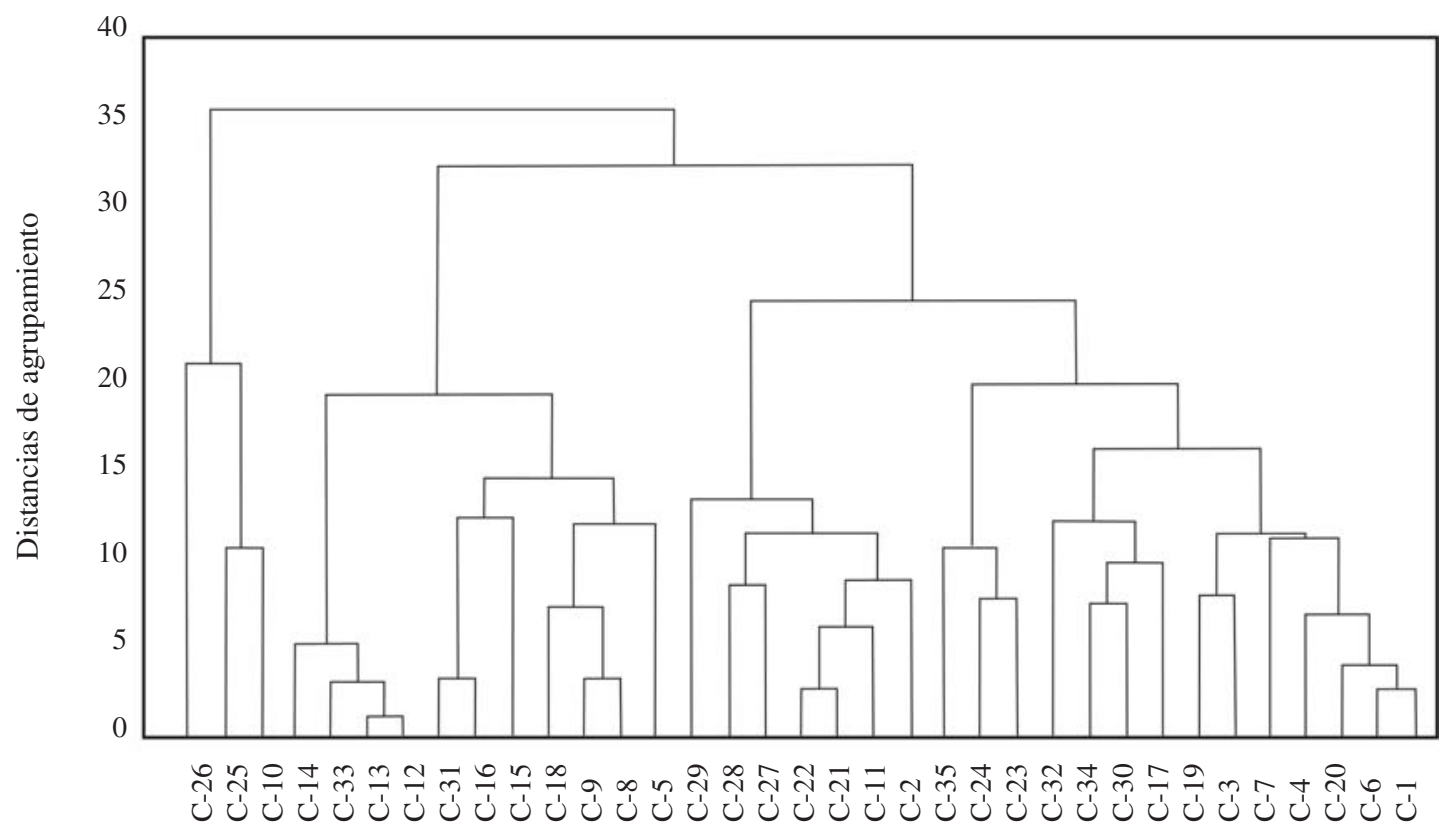

Figura 3. Análisis de agrupamientos. Pastas cerámicas de la Serie 1 ( 35 pastas x 4 variables). Cluster Analysis. Series 1 ceramic/paste (35 pastes $x 4$ variables). 


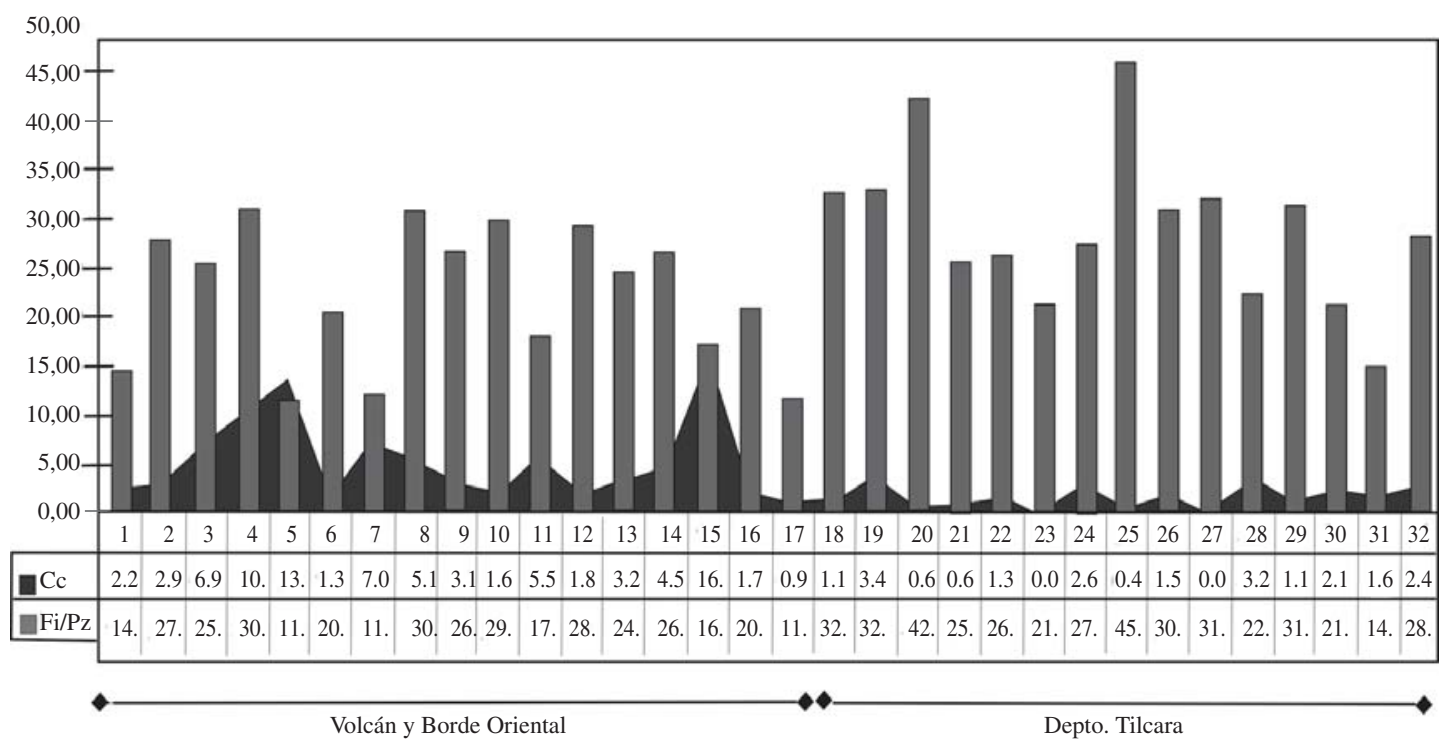

Figura 4. Porcentajes comparativos de cuarcitas y filitas/pizarras en cerámicas del sector meridional y central de la quebrada de Humahuaca. Serie 2: tipos Humahuaca y Humahuaca Inka.

Quartzite and phyllite/slate comparative percentages in ceramics from the Humahuaca Valley southern and middle portions. Serie 2: Humahuaca and Humahuaca Inka types.

$\mathrm{y}$, en coincidencia con lo observado en las pastas anteriormente comentadas, que las pastas del sector meridional ( 1 a 17$)$ se diferencian de las del sector central (18 a 32) por presentar contenidos más elevados de cuarcitas y más bajos de filitas y pizarras (Figura 4). También las primeras presentan en general contenidos más altos de cuarzo.

Por otro lado, se observaron variaciones entre las pastas de los tipos Humahuaca y Humahuaca Inka en cada sector, indicando una ausencia de control en la producción alfarera durante la dominación incaica por lo menos a nivel de selección y tratamiento de las materias primas. Por otro lado, en ninguna de las pastas analizadas se registraron litoclastos de rocas graníticas, de basalto porfírico ni tiestos molidos, una clara diferencia con las pastas analizadas de la primera serie. La ausencia de basalto porfírico y de tiesto molido en las pastas de la Serie 2 de El Cucho de Ocloyas, y la presencia de filitas y pizarras, indican que son similares a las de AP1, La Bolsa y Pucara de Volcán. Ello, sumado a la baja proporción de vasijas decoradas en Negro sobre Rojo en El Cucho de Ocloyas, plantea que las mismas no fueran elaboradas en esta guarnición de frontera, sino llevadas probablemente desde los sitios mencionados.

\section{Discusión}

Los análisis petrográficos mostraron comportamientos diferenciales de manufactura (quebrada Humahuaca meridional) en las alfarerías locales del Pucara de Volcán y de los sitios del borde oriental con respecto a los sitios del Departamento de Tilcara (sector central de la quebrada de Humahuaca), evidenciados en las variaciones porcentuales de las inclusiones no plásticas, granulometría y cocción. Las primeras reflejadas en los agrupamientos obtenidos mediante cluster analysis.

La gran mayoría de las vasijas locales del $\mathrm{Pu}$ cara de Volcán y de los sitios del borde oriental presentan pastas con porcentajes de cuarzo y cuarcitas muy superiores a las pastas del sector central, a lo que se agrega en los tipos Ordinarios y ACHI la presencia de litoclastos de roca granítica. Además, la mayoría de las pastas son poco compactas, parcialmente oxidadas y de textura gruesa no sólo en las vasijas ordinarias, sino también en las decoradas en Negro sobre Rojo, pucos Poma Negro sobre Rojo y pucos con interior Negro Pulido. Asimismo, y en relación con lo anterior, la abundancia de cuarcita y cuarzo, así como de granito de granulometría arena gruesa en los tipos Ordinarios, 
Alisados y Angosto Chico Inciso del Pucara de Volcán y del borde oriental, indica que corresponden a una misma tradición tecnológica que, para el sector sur de la quebrada de Humahuaca y para los valles sudorientales, estamos registrando desde el período Formativo (tradición San Francisco).

Un caso particular presenta la guarnición de frontera El Cucho de Ocloyas. Los conjuntos cerámicos recuperados en las excavaciones muestran una baja representación de cerámicas de origen quebradeño (Negro sobre Rojo, pucos interior Negro Pulido y ausencia de pucos Poma Negro sobre Rojo) y una alta incidencia de fragmentos Corrugados complejos, Unguiculados e impresos por cordelería que caracterizan a la alfarería de tradición chaqueña. Los fragmentos con improntas de cordelería están ausentes en todos los otros sitios y tampoco existen referencias para enclaves de la frontera oriental ubicados más al norte (Nielsen 1989; Raffino 1993).

Las pastas de los fragmentos con improntas de cordelería, corrugados, unguiculados, naranjas alisados, un porcentaje importante de ordinarios, ACHI y negros pulidos, presentan inclusiones redondeadas de basalto porfírico de hasta $2 \mathrm{~mm}$, en proporciones que varían desde $1,5 \%$ a $20 \%$. Resulta evidente que en El Cucho de Ocloyas existe un componente de alfarerías de tradición chaqueña que pudieron ser manufacturadas en el sitio mismo o traídas de áreas orientales ubicadas dentro del mismo ámbito de las sierras subandinas. El lugar más distante donde afloran estos basaltos es la serranía de Santa Bárbara, $70 \mathrm{~km}$ al este de El Cucho de Ocloyas. En AP1 y La Bolsa son prácticamente inexistentes las pastas cerámicas con basalto porfírico. Se trataría de vasijas procedentes de la frontera oriental, ya que ninguna cerámica local de Volcán presenta estas inclusiones no plásticas (Cremonte 2001; Cremonte et al. 1999). Por el contrario, los fragmentos pintados en Negro sobre Rojo, que presentan abundantes inclusiones de filitas y pizarras, corresponderían a vasijas de sitios más occidentales como, por ejemplo, AP1 o del sector sur de la quebrada de Humahuaca, basándonos en las similitudes texturales de las pastas. Las lutitas y pizarras que presentan estas pastas proceden de la Formación Puncoviscana que se encuentra a lo largo de la quebrada de Humahuaca (en la cordillera oriental), pero distante a más de $10 \mathrm{~km}$ del lugar donde se emplaza El Cucho de Ocloyas.
Los conjuntos cerámicos muestran, asimismo, otras diferencias a nivel de atributos morfológicos y decorativos, que se suman a las de las pastas comentadas en este trabajo. Para los Desarrollos Regionales, en el sector meridional el porcentaje de vasijas decoradas en Negro sobre Rojo es notoriamente más bajo que en el sector central y predominan las superficies alisadas, así como los motivos en línea media a gruesa (> $4 \mathrm{~mm})$. En un estudio comparativo entre las cerámicas del Pucara de Volcán y las del Pucara de Tilcara pudo registrarse que en el primero la simetría rotacional era mucho más común en la decoración interna de los pucos y escudillas y que era mayor la variedad de motivos reticulados, pero con una menor cantidad de elementos. La banda horizontal reticulada de líneas gruesas es el motivo más frecuente, sobre todo, en el interior de escudillas evertidas de paredes rectas. Esta forma de vasijas abiertas es predominante en Pucara de Volcán y no las hemisféricas medianas a grandes con asas como ocurre en el Pucara de Tilcara (Cremonte et al. 1997).

Los pucos Poma Negro sobre Rojo quebradeños son casi excepcionales en el sector meridional y presentan variaciones en la manufactura y en la decoración. La baja frecuencia de estos pucos en Pucara de Volcán ha sido confirmada en las excavaciones realizadas recientemente.

Las ollas Angosto Chico Inciso y los Corrugados son notoriamente más comunes en el sector meridional y las primeras presentan mayor variación morfológica y decorativa. Si bien las ollas ACHI han sido de manufactura local en cada sector, en Pucara de Volcán y en el borde oriental las pastas son diferentes y conforman un mismo grupo con las de vasijas ordinarias y alisadas sin decoración. Otras diferencias se basan en la gran cantidad de fragmentos de vasijas ordinarias con paredes externas "peinadas", así como la presencia de "revoque o barbotina". También es común que el engobe rojo de las vasijas pintadas no esté bien adherido a la pared (desprendiéndose fácilmente al tacto).

En la segunda mitad del siglo XIII y en relación con los procesos de concentración demográfica e intensificación productiva que llevaron a un nuevo orden social estratificado y consolidado alrededor del siglo XIV (Nielsen 2001), se desarrolla un estilo cerámico emblemático de los señoríos que ocuparon la quebrada de Humahuaca. Esta cerámica está caracterizada por vasijas pintadas en las que, dentro de un repertorio bastante restringi- 
do de formas y diseños, los motivos geométricos negros contrastan, agresivamente, sobre un fondo rojo. A pesar de su gran uniformidad, el estudio comparativo de conjuntos alfareros permitió detectar variaciones locales entre las unidades sociopolíticas que se desarrollaron en el centro y sur de la quebrada. Estas variaciones se manifiestan, por ejemplo, en algunos comportamientos de manufactura (características de las materias primas agregadas como material antiplástico condiciones de cocción, tratamientos y acabados de las superficies), así como en el consumo diferencial de algunos tipos y formas de vasijas.

La quebrada de Humahuaca presenta importantes vías de comunicación (tanto con las tierras altas occidentales como con las orientales) que facilitaron la participación de las sociedades locales en esferas de interacción fuera de sus límites. En este sentido, el sur de la Quebrada de Humahuaca, donde se emplaza el Pucara de Volcán y las yungas de su borde oriental (más húmedas y templadas), aportarían una oferta de recursos económicos más rica y diferente respecto de la del área central del valle. A ello se suman la proximidad y facilidad de acceso que a esta latitud existe entre la puna occidental, el fondo de valle y las yungas de Tiraxi. Estas situaciones pudieron incidir fuertemente en la estrategia de ocupación y control de esta región ejercida durante la dominación incaica (Cremonte y Garay de Fumagalli 1997).

Además, el gran poblado prehispánico de Volcán muestra un patrón arquitectónico que no es característico en la quebrada de Humahuaca. Sus rasgos más notorios son la absoluta predominancia de recintos con sus cuatro ángulos redondeados y los pisos semisubterráneos de las habitaciones. La primera de estas características se reitera en los poblados tardíos de la quebrada del Toro (p.ej., Tastil, Morohuasi) y del valle Calchaquí medio. Mientras que en el centro y norte de la quebrada de Humahuaca son típicas las esquinas en ángulo recto. Tampoco existen similitudes en las prácticas mortuorias. Como ya se dijo, en el área residencial del Pucara de Volcán, los muertos fueron fundamentalmente inhumados en el patio de las unidades domésticas. En los sitios tardíos del Departamento Tilcara, y como ocurre en el sitio homónimo "Pukara de Tilcara", las inhumaciones en el área residencial se realizaron por debajo del piso de recintos más pequeños de las viviendas.
Sin duda, para comprender la estructuración étnica de las unidades sociales del pasado es necesario diseñar y aplicar una metodología precisa para el estudio de variados y completos contextos. Los registros arqueológicos y en nuestro caso, por cierto, incompletos, permiten detectar diferencias en la cultura material, manifestadas en diversas dimensiones, pero de ningún modo pretendemos plantear identificaciones étnicas ni posicionarnos desde una postura objetivista o subjetivista de la etnicidad (Jones 1995:5). Sin embargo, mediante el análisis de los registros arqueológicos podemos pretender acercarnos a la identidad de un grupo, intentando detectar e interpretar algunos componentes de la misma (a través de distinciones sociales o jerárquicas, de interacción, de consumo diferencial de bienes, de uso del espacio, etc).

Desde esta perspectiva es que encaramos el estudio de aspectos tecnológicos de la cerámica como una aproximación poco explorada en los estudios de identidad (Cremonte 2001:99). La dimensión tecnológica no sólo está referida a las prácticas estrictamente tecnológicas, sino que también abarca toda la gama de información necesaria para las mismas, así como comportamientos sociales asociados. Si consideramos la noción de cultura de Geertz (1973) como un patrón históricamente transmitido de significados, por medio de los cuales los seres humanos se comunican, perpetúan y desarrollan sus conocimientos y actitudes hacia la vida, lo que denominamos cultura material está directamente involucrada en las prácticas sociales de transmisión de conocimiento y en los comportamientos. Mediante la cultura material podemos identificar o caracterizar la cultura de un grupo y, a partir de allí, tener puntos de referencia sobre la identidad social o grupal.

Como resultado del estudio realizado en el sector sur de la quebrada de Humahuaca y su borde oriental, y en sitios del sector central (Departamento Tilcara) detectamos idiosincrasias locales en la manufactura y distribución cerámica, que habrían contribuido en la construcción de las identidades grupales. Estas idiosincrasias como expresiones diferenciales de la "materialidad" resultarían de un conjunto de variables, dentro de las cuales privilegiamos a las siguientes: (a) la presencia de componentes tradicionales en la zona sur y oriental correspondientes a la Tradición o Complejo Cerámico San Francisco del período Formativo; (b) diferentes procesos de interacción que para la zona sur y 
oriental se reflejan en contactos con el borde occidental de la Puna salteña (cabeceras de la quebrada del Toro); y (c) la incorporación selectiva de pautas "transmitidas" a través de un estilo regional que, para los períodos de los Desarrollos Regionales e Incaico, se impone en la producción cerámica de la quebrada de Humahuaca, caracterizado por las vasijas pintadas en Negro sobre Rojo con motivos geométricos simples y otros tipos asociados contribuyen a delinear una frontera social dentro de la región Humahuaca y que abarca también su borde oriental.

Finalmente nos preguntamos si las idiosincrasias materiales, como las comentadas en este trabajo, reflejan diferentes historias locales e identidades que han conformado ese mosaico tradicionalmente homogeneizado como "Cultura Humahuaca". En este sentido y como hipótesis de trabajo planteamos la probable existencia de una frontera interna entre los sectores central y suroriental de la quebrada de Humahuaca, y que la misma se correlacionaría con la de los territorios políticos de los Tilianes (sector sur) y la de los Tilcara (sector central) citados en algunas fuentes etnohistóricas (Salas 1993[1945]; Sica y Sánchez 1996) como los descendientes de antiguos señoríos quebradeños.

Agradecimientos: Esta investigación fue realizada en el marco de los Proyectos PIP-CONICET 02670, PICT 08720 y SECTER (UNJu) E009. Las secciones delgadas fueron realizadas en el taller de petrotomía del Instituto de Geología y Minería (UNJu), y agradezco a la geóloga Alba Díaz la colaboración brindada. Así como a Gabriel Lamas por la preparación de las ilustraciones y a los revisores anónimos de Chungara por sus atentas sugerencias.

\section{Referencias Citadas}

Cocilovo, J., H. Varela y S. Valdano

2001 Estructura de la población antigua de la Quebrada de Humahuaca. En Historia Argentina Prehispánica, editado por E. Berberián y A. Nielsen, Tomo I, pp. 265-288. Editorial Brujas, Córdoba.

Cremonte, B.

2001 Las pastas cerámicas como una contribución a los estudios de identidad. Actas del XIII Congreso Nacional de Arqueología Argentina. Tomo 1:99-210, Córdoba.

Cremonte, B. y M. Garay de Fumagalli

1997 El pukara de Volcán en el sur de la Quebrada de Humahuaca. ¿Un eje articulador de las relaciones entre las Yungas y las Tierras Altas? (Provincia de Jujuy, Argentina). Estudios Atacameños 14:175-188.

1998 Correlación cronológica del yacimiento de Volcán con sitios de los Valles Orientales (Sector Meridional-Quebrada de Humahuaca). Avances en Arqueología 3:191-212.

Cremonte, B., G. Ortiz y A. Delgado

1997 Las vasijas en los estantes. Clasificación de una colección descontextualizada. Cuadernos 9:9-30.

Cremonte, B. y N. Solís

1998 La cerámica del Pucara de Volcán: Variaciones locales y evidencias de interacción. En Los Desarrollos Locales y sus Territorios, editado por B. Cremonte, pp. 155-196. Ediciones Universidad Nacional de Jujuy, Jujuy.

Cremonte, B., N. Solís y L. Botto.

1999 Materias primas empleadas en la manufactura cerámica en la Quebrada de Humahuaca (Dto. Tumbaya y Dto. Tilcara). En Los Tres Reinos: Prácticas de Recolección en el Cono Sur de América, editado por C. Aschero, A. Korstanje y P. Vuoto, pp.15-25. Universidad Nacional de Tucumán, Tucumán.
Garay de Fumagalli, M.

1998 El Pucara de Volcán, historia ocupacional y patrón de instalación. En Los Desarrollos Locales y sus Territorios, editado por B. Cremonte, pp. 131-153. Ediciones Universidad Nacional de Jujuy, Jujuy.

Geertz, C.

1973 The Interpretation of Cultures. Basic Books Inc., New York.

Jones, S.

1995 The Archaeology of Ethnicity. Constructing Identities in the Past and Present. Tesis Doctoral, Department of Archaeology, University of Southampton.

Nielsen, A.

1989 La Ocupación Indígena del Territorio Humahuaca Oriental Durante los Períodos de Desarrollos Regionales e Inka. Tesis Doctoral, Universidad Nacional de Córdoba, Córdoba.

2001 Evolución social en la Quebrada de Humahuaca. (AD 700-1.536). En Historia Argentina Prehispánica, editado por E. Berberián y A. Nielsen, Tomo 1, pp. 17-264. Editorial Brujas, Córdoba.

Raffino, R., editor

1993 Inka. Arqueología, Historia y Urbanismo del Altiplano Andino. Ediciones Corregidor, Buenos Aires.

Salas, A.

1993 [1945] El Antigal de Ciénaga Grande. Edición conjunta Universidad Nacional de Jujuy e Instituto Interdisciplinario Tilcara, Jujuy.

Sica, G. y S. Sánchez

1996 Pueblos indígenas de la Quebrada de Humahuaca. Siglos XVI y XVII. La integración Surandina cinco siglos después. Estudios y Debates Regionales Andinos 91:287-306. 
